\title{
Effect on productivity of silk (Antheraea mylitta) due to changes Environmental Factors in Ambikapur Surguja District CG
}

\author{
${ }^{1}$ Meena Singh, ${ }^{2}$ Dr.Manoj Singh \\ Kalinga University Kotani, \\ New Raipur, 492002, Chhattisgarh, India
}

\begin{abstract}
Ambikapur is a city is surguja district of Chhattisgarh. The oldest districts of the Indian state of Chhattisgarh in the east.Central Indian Ambikapur is also the divisional headquarters of surguja district which consists of the five districts of surguja, koriya, balrampur, surajpur and jashpur. Ambikapur is located at $23^{\circ} 37^{\prime} 25^{\prime}$ 'to $24^{\circ}$ 6' $^{\prime 2} 7^{\prime}$ 'North latitude and $81^{\circ} 34^{\prime} 40^{\prime \prime}$ to $84^{\circ} 4^{\prime} 40^{\prime}$ " east longitude, $244.62 \mathrm{~km}$ long east to westand 167.37 broad north to south. This land has as area of about 16359 sqkm. Ambikapur Tasar silkworm areas of 10 hectares in Arjuna plants culture. In current issue changing global climate seems to be one of the major hindrance in the effect on productivity of silkworm Antheraea mylitta. Various climatic factors such as temperature, humidity, light, air, wind etc. The present experimental analysis conducted in environment factors the role and influence of temperature and humidity in the growth of pupa from larvae of silkworm Antheraea mylitta.It is also observed that weight of pupa and shell reared at temperature $22-26{ }^{\circ} \mathrm{c}$ and $80-85 \%$ realative humidity are more. The present observation of the effect on productivity of silk due to environmental factors in Ambikapur district surguja.The study includes the steps to be taken for the management of condition and improved quality and quality of silk production in future.
\end{abstract}

Keywords: Abiotic factors, temperature, humidity, rainfall, wind, diseases, pest.

- Corresponding Author

- Name:Meena Singh

- $\quad$ Phone:9131090344

- Email: nevaan.ambikapur@gmail.com

\section{Introduction}

Sericulture is the Cultivation of silk through rearing of silkworm.It is an agro-based industry.sericulture also includes the practical aspects such as increasing productivity of land as well as labour,stabilization of cocoon production improvement of silk,fabric and generating profitable income for rural poor people.The discovered in china between 2600 and 2700 BC.Today china and India are two main producer with more than $60 \%$ of the worlds.Annual production India is the second largest producer of silk in world and contributes $18 \%$ of the total world raw silk production.In India silk is available with varieties such as Mulberry,Eri,tasar and munga.Tasar silkworm are reared traditionally by the tribes of Madhya Pradesh,Bihar,and Orissa;Munga and eri silk are produced exclusively in Assam.Mulberry silk is produced extensively in the states of Karnataka,West Bengal,Jammu \& Kashmir.Sericulture or silk production is the breeding and management of silkworms for the commercial production of silk.It is an Economically important insect being a primary producer of silk.All the section of sericulture Industry viz; cultivation silkworm seed production,silk rearing,reeling and weaving of the silk and collection of by products and its processing provide a large scale employment there by a source of livelihood for the rural and tribal peopal(Gregory 1914 and srivastava 2003). Chhattisgarh state is a very high quality kosa silk production.silk way of life in Chhattisgarh has become an in separable part of Indian culture and tradition should be considered for rural management and development(Dewangan etal;2011).Presently in chhattisgarh three types of silk viz; Mulberry,Tasar culture furthermore it is practiced especially in trible belts of surguja,Raigarh,Bilaspur,Korba,Bastar district of the Chhattisgarh state Keeping in view of the above facts into consideration.The silk is preferred over all other types of fibres due to its remarkable properties like water absorbency heat ressistance dying efficiency and luster.Factor mainly influence the physiology of insects are temperature and humidity.The adaptability is quite different from those of wild silkworm;Temperature,humidity,light,wind Rainy effect on the physiology of silkworm depending upon the combination of factors and development stages affecting growth development productivity and quality of silk. The present study of silk production effect on due to changeable environmental factors is possible at the outdoor condition culture also climax included future strategies to be taken for the management best successful silk production by best environmental factor conditions. 


\section{Research Methodology:-}

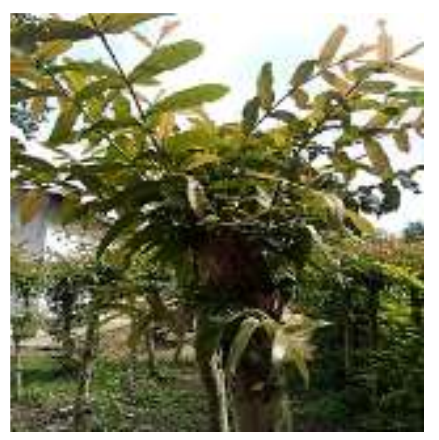

Fig 1: Terminalia arjuna food plant

Systematic position

- Kingdom - Planatae

- Phylum - Magnoliophyta

- Class - Magnoliopsida

- Order - Myrtales

- Family - Combretaceae

- Genus - Terminalia

- Species - arjuna

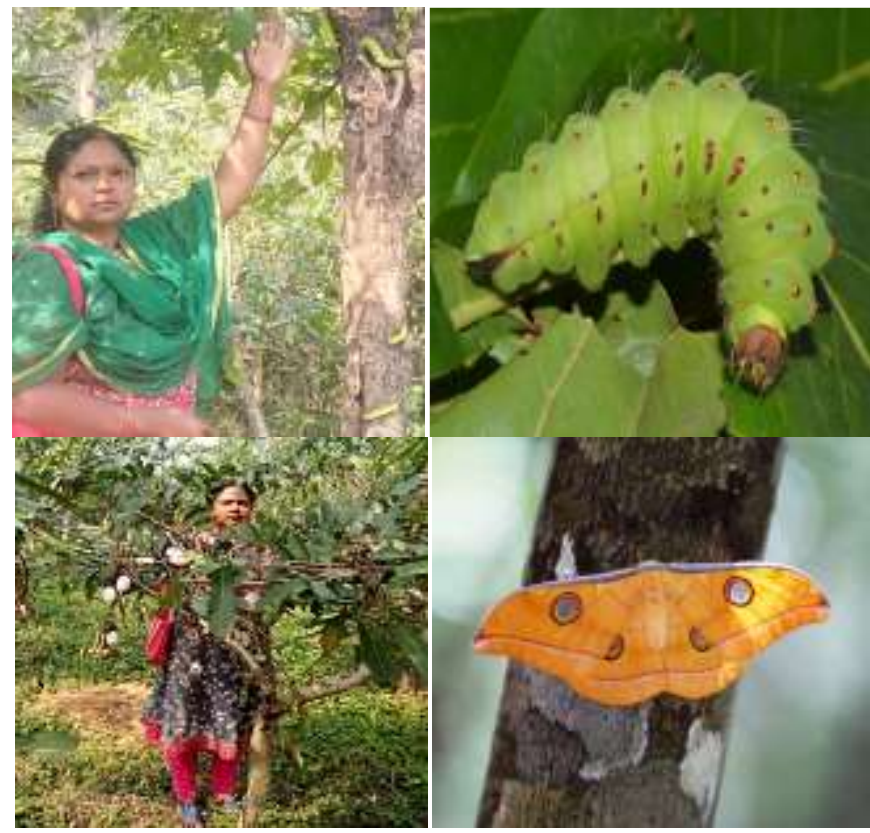

Fig 2: Antheraea Mylitta-Fifth Instar Larva, cocoon, Silkmonth

Systematic Classification:

- Kingdom

- Phylum

- Class

- Order

- Family

- Genus

- Species
Animalia
Anthropoda
Insecta
Lepidoptera
Saturniidae
Antheraea
Mylitta

The present work was sericulture center of Ambikapur Surguja district during 2018-2019.The Chhattisgarh state represented by climate is tropical.It is hot and humid because of its proximity to the tropic of cancer and its dependence on the man soons for rains.Summer temperature can reach up $45^{\circ} \mathrm{c}\left(113^{\prime} \mathrm{F}\right)$. The man soon season is from late June to October and is a welcome respite from the head.The average rainfall of Chhattisgarh is $1292 \mathrm{~mm}$. Winter is from November to January and is a good time to visit 
Chhattisgarh winters are pleasant with low temperature and less humidity.Temperature increases from march to June but by the end of June,the temperature decreases as the mansoon moved.In July Temperature remain about $27^{0} \mathrm{c}-27^{0} \mathrm{c}$, there is no variation in the temperature in the month of September and October,but in the most of September and October,when the sky is clear,there is a slight increase in the temperature.There are 3 seasons in the Chhattisgarh.

1.Hot season - this season starts from march and goes up to mid june.In the month of June when the sun shines vertically over the tropic of cancer; due to high temperature.The pressure decreases and temperature rises to as much as $42^{\circ} 5$ 'c in some parts of the state.

2.Rainy season - From mid June to September Ambikapur,Bilaspur and Bastar experiences rain more than 100 c.m.

3.Winter season - It starts from November to January.It September Temperature starts decreasing this is caused due to rainfall and humidity and winter arrives in November.

The Surguja district enjoy tropical climate which characterized by a hot summer and well distributed rainfall.The present work was in Ambikapur Tasar Silkworm host plant Terminalia arjuna was selected for the study.The period of sampling will collected from sericulture center of Ambikapur was $1^{\text {st }}$ week of July 2018,total areas covered under Tasar fields is about 10 hecters through effective area is about 8 hecters and selected to $1^{\text {st }}$ crop 50 Trees (2 plot) in Terminalia arjuna.Each plots consisted of approximately 25 trees. At First Tasar silkworm eggs has been taken at $10 \mathrm{dfl}$ after that having keeping it in tray,then larves began to come out. After being observation of environmental factors,temperature,humidity,speed of wind.Then processes of making of cocoon is observed.After that doing analysis of cocoons production is done.After this crop is totally prepared.All the eggs have been taken in equal quantity.In these three crops 2018-2019,the whole process is completed.After obserningof these one year we know the maximum production of silk.All these environmental factor effects of production capacity and they determine the quantity of silk

\section{$\underline{\text { 1st Crop - Rearing Performance }}$}

\begin{tabular}{|c|c|c|c|c|c|c|c|c|c|c|c|}
\hline \multirow[t]{2}{*}{ 3-Jul-18 } & \multirow{2}{*}{$\begin{array}{l}12 \text {-Aug- } \\
18 \\
\text { Date }\end{array}$} & \multicolumn{10}{|c|}{$\begin{array}{c}\text { Meteorological Data (Day-wise) at Ambikapur Antheraea mylitta Feed in Arjuna Plant } \\
2018-19\end{array}$} \\
\hline & & T.MAX & T.MIN & $\begin{array}{l}\mathbf{R} \\
\text { Fall } \\
(\mathbf{m m})\end{array}$ & $\begin{array}{l}\text { RH- } \\
\text { I }\end{array}$ & $\begin{array}{l}\text { RH- } \\
\text { II }\end{array}$ & $\begin{array}{l}\text { W } \\
\text { SPEED }\end{array}$ & $\begin{array}{l}\text { Rainy } \\
\text { Day }\end{array}$ & $\begin{array}{l}\text { No. of } \\
\text { larva }\end{array}$ & Mor.No. & Mor.\% \\
\hline & 1-Jul-18 & 31.2 & 24.2 & 0.0 & 83 & 58 & 4.7 & & & & \\
\hline & 2-Jul-18 & 31.4 & 21.2 & 61.0 & 98 & 72 & 4.5 & 1 & & & \\
\hline \multirow[t]{7}{*}{ 1st crop } & 3-Jul-18 & 30.0 & 21.4 & 4.8 & 90 & 74 & 4.2 & 1 & & & \\
\hline & 4-Jul-18 & 29.2 & 21.1 & 10.3 & 95 & 73 & 6.8 & 1 & & & \\
\hline & 5-Jul-18 & 29.5 & 23.5 & 0.0 & 84 & 67 & 7.3 & & & & \\
\hline & 6-Jul-18 & 30.5 & 23.6 & 0.0 & 84 & 52 & 3.7 & & & & \\
\hline & 7-Jul-18 & 34.0 & 21.5 & 39.4 & 98 & 59 & 3.5 & 1 & & & \\
\hline & 8-Jul-18 & 33.0 & 25.0 & 0.0 & 85 & 59 & 1.6 & & & & \\
\hline & 9-Jul-18 & 33.5 & 24.5 & 0.0 & 88 & 74 & 2.9 & & & & \\
\hline \multirow[t]{6}{*}{ 1st larva* } & 10-Jul-18 & 31.2 & 22.9 & 44.0 & 98 & 68 & 2.8 & 1 & 1800 & 140 & 7.8 \\
\hline & 11-Jul-18 & 31.2 & 23.7 & 0.0 & 92 & 66 & 2.3 & & & & \\
\hline & 12-Jul-18 & 31.7 & 24.7 & 0.4 & 94 & 84 & 2.7 & & & & \\
\hline & 13-Jul-18 & 29.5 & 24.5 & 2.3 & 94 & 76 & 1.4 & & & & \\
\hline & 14-Jul-18 & 30.2 & 23.2 & 15.3 & 95 & 77 & 2.7 & 1 & & & \\
\hline & 15-Jul-18 & 29.0 & 24.4 & 4.2 & 94 & 66 & 1.7 & 1 & & & \\
\hline \multirow[t]{5}{*}{ 2ndlarva* } & 16-Jul-18 & 31.7 & 23.8 & 6.2 & 97 & 70 & 3.9 & 1 & 1660 & 125 & 7.5 \\
\hline & 17-Jul-18 & 30.5 & 23.5 & 23.7 & 95 & 91 & 4.4 & 1 & & & \\
\hline & 18-Jul-18 & 27.5 & 23.5 & 44.8 & 98 & 98 & 4.3 & 1 & & & \\
\hline & 19-Jul-18 & 25.0 & 22.6 & 24.7 & 98 & 84 & 3.7 & 1 & & & \\
\hline & 20-Jul-18 & 28.0 & 23.2 & 30.0 & 98 & 71 & 1.9 & 1 & & & \\
\hline \multirow[t]{6}{*}{ 3rd larva* } & 21-Jul-18 & 31.6 & 21.5 & 19.8 & 98 & 80 & 3.2 & 1 & 1535 & 60 & 3.9 \\
\hline & 22-Jul-18 & 30.3 & 23.0 & 0.2 & 93 & 100 & 3.2 & & & & \\
\hline & 23-Jul-18 & 25.0 & 23.0 & 39.6 & 98 & 87 & 4.5 & 1 & & & \\
\hline & 24-Jul-18 & 26.5 & 22.6 & 0.0 & 92 & 79 & 11.0 & & & & \\
\hline & 25-Jul-18 & 27.6 & 22.5 & 15.4 & 97 & 92 & 9.8 & 1 & & & \\
\hline & 26-Jul-18 & 25.2 & 22.1 & 32.4 & 100 & 98 & 7.9 & 1 & & & \\
\hline \multirow[t]{2}{*}{ 4th larva* } & 27-Jul-18 & 24.6 & 21.0 & 6.6 & 98 & 89 & 3.6 & 1 & 1475 & 52 & 3.5 \\
\hline & 28-Jul-18 & 26.0 & 22.2 & 4.4 & 90 & 73 & 7.4 & 1 & & & \\
\hline
\end{tabular}




\begin{tabular}{|c|c|c|c|c|c|c|c|c|c|c|c|}
\hline & 29-Jul-18 & 28.5 & 22.2 & 3.7 & 98 & 98 & 4.7 & 1 & & & \\
\hline & 30-Jul-18 & 25.0 & 22.1 & 22.2 & 95 & 75 & 3.6 & 1 & & & \\
\hline & 31-Jul-18 & 28.5 & 22.5 & 0.0 & 86 & 87 & 4.4 & & & & \\
\hline & 1-Aug-18 & 26.0 & 21.4 & 0.4 & 92 & 86 & 6.6 & & & & \\
\hline & 2-Aug-18 & 25.2 & 20.8 & 10.0 & 98 & 86 & 7.9 & 1 & & & \\
\hline \multirow[t]{10}{*}{ 5th larva* } & 3-Aug-18 & 26.0 & 23.2 & 0.1 & 86 & 79 & 4.5 & & 1423 & 11 & 0.8 \\
\hline & 4-Aug-18 & 27.6 & 22.5 & 0.0 & 89 & 67 & 3.4 & & & & \\
\hline & 5-Aug-18 & 30.5 & 23.5 & 5.0 & 87 & 57 & 2.6 & 1 & & & \\
\hline & 6-Aug-18 & 30.0 & 23.2 & 0.0 & 93 & 78 & 1.8 & & & & \\
\hline & 7-Aug-18 & 27.5 & 21.5 & 23.8 & 98 & 100 & 2.2 & 1 & & & \\
\hline & 8-Aug-18 & 25.5 & 22.5 & 19.4 & 92 & 65 & 2.2 & 1 & & & \\
\hline & 9-Aug-18 & 30.2 & 22.6 & 1.2 & 83 & 89 & 5.7 & & & & \\
\hline & $\begin{array}{l}\text { 10-Aug- } \\
18\end{array}$ & 27.6 & 23.5 & 1.0 & 97 & 80 & 2.4 & & & & \\
\hline & $\begin{array}{l}\text { 11-Aug- } \\
18\end{array}$ & 29.3 & 22.5 & 11.6 & 97 & 80 & 2.2 & 1 & & & \\
\hline & $\begin{array}{l}\text { 12-Aug- } \\
18\end{array}$ & 29.6 & 23.0 & 0.0 & 92 & 73 & 2.0 & & & & \\
\hline Total & & & & & & & & & 1412 & 388 & \\
\hline Prod. $\%$ & & & & & & & & & $78.44 \%$ & & \\
\hline
\end{tabular}

\section{$\underline{2^{\text {nd }} \text { Crop - Rearing Performance }}$}

\begin{tabular}{|c|c|c|c|c|c|c|c|c|c|c|c|}
\hline \multirow[t]{2}{*}{ 3-Sep-18 } & \multirow{2}{*}{$\begin{array}{l}\text { 9-Oct-18 } \\
\text { Date }\end{array}$} & \multicolumn{10}{|c|}{$\begin{array}{l}\text { Meteorological Data (Day-wise) at Ambikapur Antheraea mylitta Feed in Arjuna Plant } \\
\text { 2018-19 }\end{array}$} \\
\hline & & $\begin{array}{l}\text { T.MA } \\
\text { X }\end{array}$ & $\begin{array}{l}\text { T.MI } \\
\mathbf{N}\end{array}$ & $\begin{array}{l}\text { R Fall } \\
(\mathbf{m m})\end{array}$ & $\begin{array}{l}\text { RH- } \\
\text { I }\end{array}$ & $\begin{array}{l}\text { RH- } \\
\text { II }\end{array}$ & $\begin{array}{l}\text { W } \\
\text { SPEE } \\
\text { D }\end{array}$ & $\begin{array}{l}\text { Rain } \\
\text { y Day }\end{array}$ & $\begin{array}{l}\text { No. of } \\
\text { larva }\end{array}$ & $\begin{array}{l}\text { Mor.No } \\
\text { - }\end{array}$ & $\begin{array}{l}\text { Mor. } \\
\%\end{array}$ \\
\hline \multirow[t]{7}{*}{$\begin{array}{l}\text { 2nd } \\
\text { crop }\end{array}$} & 3-Sep-18 & 29.2 & 21.2 & 17.6 & 93 & 86 & 4.2 & 1 & & & \\
\hline & 4-Sep-18 & 25.5 & 22.5 & 0.0 & 92 & 74 & 4.7 & & & & \\
\hline & 5-Sep-18 & 28.8 & 21.6 & 11.0 & 98 & 90 & 4.6 & 1 & & & \\
\hline & 6-Sep-18 & 25.5 & 22.2 & 22.6 & 98 & 73 & 6.8 & 1 & & & \\
\hline & 7-Sep-18 & 29.0 & 21.5 & 26.2 & 100 & 97 & 2.0 & 1 & & & \\
\hline & 8-Sep-18 & 24.2 & 20.7 & 11.4 & 93 & 75 & 3.4 & 1 & & & \\
\hline & 9-Sep-18 & 27.0 & 21.9 & 0.0 & 95 & 84 & 3.7 & & & & \\
\hline \multirow[t]{5}{*}{$\begin{array}{c}\text { 1st } \\
\text { larva* }\end{array}$} & 10-Sep-18 & 28.5 & 23.0 & 0.8 & 97 & 64 & 3.0 & & 1500 & 90 & 6 \\
\hline & 11-Sep-18 & 29.5 & 22.4 & 0.0 & 87 & 61 & 2.1 & & & & \\
\hline & $12-$ Sep-18 & 30.4 & 21.8 & 0.0 & 84 & 64 & 2.3 & & & & \\
\hline & 13-Sep-18 & 29.5 & 22.3 & 0.4 & 92 & 72 & 3.0 & & & & \\
\hline & 14-Sep-18 & 28.8 & 21.5 & 6.7 & 93 & 60 & 2.9 & 1 & & & \\
\hline \multirow[t]{4}{*}{$\begin{array}{c}\text { 2nd } \\
\text { larva* }\end{array}$} & $15-$ Sep-18 & 31.0 & 22.5 & 0.0 & 89 & 58 & 2.6 & & 1410 & 25 & 1.8 \\
\hline & 16-Sep-18 & 30.8 & 21.6 & 0.0 & 92 & 62 & 1.6 & & & & \\
\hline & 17-Sep-18 & 31.0 & 22.0 & 0.0 & 92 & 62 & 2.0 & & & & \\
\hline & 18-Sep-18 & 29.8 & 21.9 & 0.0 & 92 & 54 & 1.6 & & & & \\
\hline \multirow[t]{5}{*}{$\begin{array}{c}\text { 3rd } \\
\text { larva* }\end{array}$} & 19-Sep-18 & 32.0 & 21.5 & 0.0 & 92 & 55 & 1.4 & & 1385 & 18 & 1.3 \\
\hline & 20-Sep-18 & 31.3 & 19.8 & 0.0 & 93 & 61 & 2.0 & & & & \\
\hline & 21-Sep-18 & 29.8 & 20.5 & 1.6 & 93 & 77 & 4.2 & & & & \\
\hline & $22-$ Sep-18 & 27.2 & 20.7 & 0.0 & 84 & 67 & 6.9 & & & & \\
\hline & 23-Sep-18 & 29.6 & 20.6 & 0.0 & 80 & 60 & 4.7 & & & & \\
\hline \multirow[t]{3}{*}{$\begin{array}{c}\text { 4th } \\
\text { larva* }\end{array}$} & 24-Sep-18 & 30.2 & 20.5 & 0.0 & 88 & 63 & 3.0 & & 1367 & 10 & 0.7 \\
\hline & 25-Sep-18 & 32.0 & 22.0 & 0.0 & 90 & 56 & 3.7 & & & & \\
\hline & 26-Sep-18 & 32.0 & 20.7 & 0.0 & 92 & 48 & 2.9 & & & & \\
\hline
\end{tabular}




\begin{tabular}{|c|c|c|c|c|c|c|c|c|c|c|}
\hline & 27-Sep-18 & 31.6 & 21.0 & 0.0 & 90 & 47 & 3.5 & & & \\
\hline & 28-Sep-18 & 33.0 & 20.7 & 0.0 & 84 & 43 & 3.4 & & & \\
\hline & 29-Sep-18 & 33.2 & 19.0 & 0.0 & 77 & 49 & 1.4 & & & \\
\hline $\begin{array}{c}5 \text { th } \\
\text { larva* }\end{array}$ & 30-Sep-18 & 32.0 & 19.7 & 0.0 & 85 & 49 & 1.6 & 1357 & 7 & 0.5 \\
\hline & 1-Oct-18 & 32.0 & 18.6 & 0.0 & 80 & 39 & 1.5 & & & \\
\hline & 2-Oct-18 & 33.2 & 19.2 & 0.0 & 71 & 38 & 1.7 & & & \\
\hline & 3-Oct-18 & 33.2 & 18.7 & 0.0 & 69 & 37 & 1.8 & & & \\
\hline & 4-Oct-18 & 33.0 & 18.0 & 0.0 & 71 & 36 & 1.8 & & & \\
\hline & 5-Oct-18 & 33.0 & 19.0 & 0.0 & 81 & 37 & 1.2 & & & \\
\hline & 6-Oct-18 & 33.2 & 18.1 & 0.0 & 88 & 37 & 1.6 & & & \\
\hline & 7-Oct-18 & 32.6 & 17.8 & 0.0 & 87 & 32 & 1.3 & & & \\
\hline & 8-Oct-18 & 32.0 & 17.0 & 0.0 & 75 & 37 & 1.0 & & & \\
\hline & 9-Oct-18 & 32.5 & 15.5 & 0.0 & 76 & 34 & 0.6 & & & \\
\hline Total & & & & & & & & 1350 & 150 & \\
\hline Prod.\% & & & & & & & & $90.00 \%$ & & \\
\hline
\end{tabular}

$\underline{\text { 3rd Crop -Rearing Performance }}$

\begin{tabular}{|c|c|c|c|c|c|c|c|c|c|c|c|}
\hline \multirow[t]{2}{*}{$\begin{array}{c}5 \text {-Nov- } \\
18\end{array}$} & \multirow{2}{*}{$\begin{array}{c}\text { 31-Jan-19 } \\
\text { Date }\end{array}$} & \multicolumn{10}{|c|}{$\begin{array}{c}\text { Meteorological Data (Day-wise) at Ambikapur Antheraea mylitta Feed in Arjuna Plant } \\
2018-19\end{array}$} \\
\hline & & T.MAX & T.MIN & $\begin{array}{c}\text { R Fall } \\
(\mathbf{m m})\end{array}$ & $\begin{array}{c}\text { RH- } \\
\text { I }\end{array}$ & $\begin{array}{c}\text { RH- } \\
\text { II }\end{array}$ & $\begin{array}{c}\text { W } \\
\text { SPEED }\end{array}$ & $\begin{array}{c}\text { Rainy } \\
\text { Day }\end{array}$ & $\begin{array}{l}\text { No. of } \\
\text { larva }\end{array}$ & $\begin{array}{c}\text { Mor. } \\
\text { No. }\end{array}$ & Mor.\% \\
\hline \multirow[t]{16}{*}{$\begin{array}{c}\text { 3rd } \\
\text { Crop }\end{array}$} & 5-Nov-18 & 30.0 & 15.6 & 0.0 & 90 & 45 & 3.1 & & & & \\
\hline & 6-Nov-18 & 30.8 & 17.6 & 0.0 & 93 & 42 & 2.1 & & & & \\
\hline & 7-Nov-18 & 30.2 & 12.4 & 0.0 & 62 & 30 & 1.4 & & & & \\
\hline & 8-Nov-18 & 30.5 & 10.0 & 0.0 & 93 & 29 & 1.6 & & & & \\
\hline & 9-Nov-18 & 28.4 & 9.0 & 0.0 & 84 & 28 & 1.3 & & & & \\
\hline & 10-Nov-18 & 28.6 & 9.0 & 0.0 & 95 & 34 & 1.6 & & & & \\
\hline & 11-Nov-18 & 27.8 & 9.1 & 0.0 & 95 & 34 & 1.4 & & & & \\
\hline & 12-Nov-18 & 26.9 & 9.0 & 0.0 & 95 & 31 & 1.4 & & & & \\
\hline & 13-Nov-18 & 27.0 & 10.5 & 0.0 & 86 & 29 & 2.0 & & & & \\
\hline & 14-Nov-18 & 28.5 & 11.0 & 0.0 & 89 & 26 & 1.6 & & & & \\
\hline & 15-Nov-18 & 30.0 & 12.5 & 0.0 & 81 & 31 & 1.7 & & & & \\
\hline & 16-Nov-18 & 29.8 & 13.3 & 0.0 & 74 & 29 & 2.0 & & & & \\
\hline & 17-Nov-18 & 29.5 & 12.3 & 0.0 & 91 & 33 & 3.1 & & & & \\
\hline & 18-Nov-18 & 27.2 & 10.5 & 0.0 & 86 & 26 & 1.6 & & & & \\
\hline & 19-Nov-18 & 28.0 & 10.0 & 0.0 & 91 & 35 & 1.3 & & & & \\
\hline & 20-Nov-18 & 29.0 & 11.0 & 0.0 & 87 & 25 & 1.5 & & & & \\
\hline \multirow[t]{15}{*}{$\begin{array}{c}1 \text { st } \\
\text { larva* }\end{array}$} & 21-Nov-18 & 29.8 & 10.5 & 0.0 & 91 & 30 & 1.0 & & 1680 & 300 & 17.85 \\
\hline & 22-Nov-18 & 29.0 & 9.9 & 0.0 & 91 & 32 & 1.9 & & & & \\
\hline & 23-Nov-18 & 27.0 & 7.8 & 0.0 & 90 & 32 & 1.9 & & & & \\
\hline & 24-Nov-18 & 27.2 & 8.0 & 0.0 & 93 & 31 & 1.6 & & & & \\
\hline & 25 -Nov-18 & 25.0 & 7.0 & 0.0 & 95 & 34 & 1.6 & & & & \\
\hline & 26-Nov-18 & 25.5 & 7.3 & 0.0 & 92 & 32 & 1.8 & & & & \\
\hline & 27-Nov-18 & 25.3 & 8.3 & 0.0 & 93 & 38 & 1.2 & & & & \\
\hline & 28-Nov-18 & 27.0 & 11.3 & 0.0 & 87 & 44 & 1.2 & & & & \\
\hline & 29-Nov-18 & 26.5 & 10.8 & 0.0 & 93 & 43 & 1.5 & & & & \\
\hline & 30-Nov-18 & 26.2 & 10.0 & 0.0 & 89 & 43 & 1.2 & & & & \\
\hline & 1-Dec-18 & 25.5 & 9.2 & 0.0 & 93 & 34 & 1.0 & & & & \\
\hline & 2-Dec-18 & 24.1 & 7.6 & 0.0 & 97 & 40 & 1.1 & & & & \\
\hline & 3-Dec-18 & 24.2 & 8.5 & 0.0 & 98 & 34 & 1.0 & & & & \\
\hline & 4-Dec-18 & 25.0 & 8.6 & 0.0 & 93 & 41 & 0.9 & & & & \\
\hline & 5-Dec-18 & 24.1 & 10.0 & 0.0 & 85 & 43 & 1.0 & & & & \\
\hline
\end{tabular}




\begin{tabular}{|c|c|c|c|c|c|c|c|c|c|c|c|}
\hline & 6-Dec-18 & 23.9 & 8.0 & 0.0 & 98 & 38 & 0.7 & & & & \\
\hline & 7-Dec-18 & 25.0 & 7.7 & 0.0 & 93 & 32 & 1.4 & & & & \\
\hline & 8-Dec-18 & 24.2 & 8.7 & 0.0 & 72 & 39 & 1.6 & & & & \\
\hline & 9-Dec-18 & 24.9 & 6.0 & 0.0 & 92 & 30 & 1.1 & & & & \\
\hline \multirow{14}{*}{$\begin{array}{c}\text { 2nd } \\
\text { larva* }\end{array}$} & 10-Dec-18 & 25.5 & 9.0 & 0.0 & 86 & 49 & 1.0 & & 1380 & 195 & 14.13 \\
\hline & 11-Dec-18 & 25.5 & 13.1 & 1.2 & 83 & 58 & 1.4 & & & & \\
\hline & 12-Dec-18 & 25.0 & 11.0 & 0.0 & 89 & 49 & 1.1 & & & & \\
\hline & 13-Dec-18 & 24.2 & 9.2 & 0.0 & 91 & 32 & 0.8 & & & & \\
\hline & 14-Dec-18 & 25.8 & 12.0 & 0.0 & 93 & 33 & 1.1 & & & & \\
\hline & 15-Dec-18 & 23.8 & 7.0 & 0.0 & 95 & 39 & 2.2 & & & & \\
\hline & 16-Dec-18 & 22.0 & 6.0 & 0.0 & 93 & 36 & 1.8 & & & & \\
\hline & 17-Dec-18 & 22.0 & 11.6 & 0.5 & 95 & 98 & 1.6 & & & & \\
\hline & 18-Dec-18 & 15.0 & 11.0 & 40.8 & 93 & 88 & 3.5 & 1 & & & \\
\hline & 19-Dec-18 & 16.0 & 7.5 & 0.0 & 87 & 57 & 1.0 & & & & \\
\hline & 20-Dec-18 & 20.0 & 6.6 & 0.0 & 92 & 44 & 1.2 & & & & \\
\hline & 21-Dec-18 & 21.0 & 5.3 & 0.0 & 84 & 39 & 1.1 & & & & \\
\hline & 22-Dec-18 & 20.0 & 3.0 & 0.0 & 91 & 30 & 1.2 & & & & \\
\hline & 23-Dec-18 & 20.5 & 4.6 & 0.0 & 94 & 33 & 1.2 & & & & \\
\hline \multirow{8}{*}{$\begin{array}{c}\text { 3rd } \\
\text { larva* }\end{array}$} & 24-Dec-18 & 21.5 & 5.4 & 0.0 & 91 & 39 & 1.0 & & 1195 & 196 & 16.54 \\
\hline & 25-Dec-18 & 21.0 & 5.7 & 0.0 & 89 & 29 & 1.0 & & & & \\
\hline & 26-Dec-18 & 20.5 & 5.2 & 0.0 & 94 & 36 & 1.4 & & & & \\
\hline & 27-Dec-18 & 21.6 & 7.6 & 0.0 & 90 & 36 & 1.0 & & & & \\
\hline & 28-Dec-18 & 22.0 & 5.2 & 0.0 & 89 & 28 & 1.9 & & & & \\
\hline & 29-Dec-18 & 19.5 & 4.2 & 0.0 & 97 & 25 & 1.6 & & & & \\
\hline & 30-Dec-18 & 20.0 & 2.6 & 0.0 & 84 & 29 & 1.1 & & & & \\
\hline & 31-Dec-18 & 19.5 & 2.0 & 0.0 & 97 & 38 & 1.0 & & & & \\
\hline $\begin{array}{c}\text { Week } \\
\text { No. }\end{array}$ & January & & & & & & & & & & \\
\hline 1 & 4th larva* & 23.2 & 5.1 & $\mathbf{0}$ & 93.0 & 29.7 & 1.1 & $\mathbf{0}$ & 989 & 79 & 7.98 \\
\hline 2 & & 22.7 & 6.8 & 0.0 & 93.0 & 41.0 & 1.6 & 0.0 & & & \\
\hline 3 & 5th larva* & 23.3 & 5.7 & 0.0 & 87.7 & 23.6 & 1.2 & 0.0 & 910 & 10 & 1.09 \\
\hline 4 & & 22.8 & 11.5 & 16.2 & 85.3 & 59.7 & 2.6 & 2.0 & & & \\
\hline \multirow[t]{3}{*}{5} & & 22.8 & 7.6 & 0.0 & 85.9 & 31.0 & 1.7 & 0.0 & & & \\
\hline & Total & & & & & & & & 900 & 780 & \\
\hline & Prod. $\%$ & & & & & & & & $53.57 \%$ & & \\
\hline
\end{tabular}

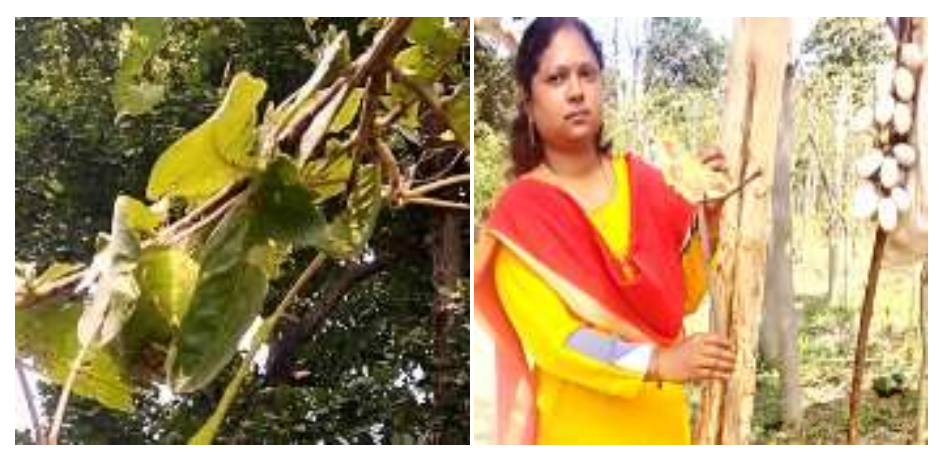

Fig 3. Spinning cocoon and cocoon,Silkmonth( Tasar silkworm) 
$1^{\text {st }}, 2^{\text {nd }}, 3^{\text {rd }}$ Crop- Cocoon weight, table $(1,2,3)$

Table 1

\begin{tabular}{|c|c|c|}
\hline \multirow{2}{*}{$\begin{array}{c}\text { Set. } \\
\text { No. }\end{array}$} & \multicolumn{2}{|c|}{$\begin{array}{c}\text { Terminalia arjuna plant } \\
\text { Cocoon Wt. in gram }\end{array}$} \\
\cline { 2 - 3 } & Female & Male \\
\hline 1 & 12.17 & 11.08 \\
\hline 2 & 10.87 & 8.55 \\
\hline 3 & 11.71 & 7.28 \\
\hline Total & 35.29 & 26.91 \\
\hline Mean & 11.76 & 8.97 \\
\hline SD & 0.751 & 1.579 \\
\hline SE & 0.433 & 0.911 \\
\hline
\end{tabular}

Table 2

\begin{tabular}{|c|c|c|}
\hline \multirow{2}{*}{$\begin{array}{c}\text { Set. } \\
\text { No. }\end{array}$} & \multicolumn{2}{|c|}{$\begin{array}{c}\text { Terminalia arjuna } \\
\text { plant Cocoon Wt. in } \\
\text { gram }\end{array}$} \\
\cline { 2 - 3 } & Female & Male \\
\hline 1 & 11.70 & 10.8 \\
\hline 2 & 12.83 & 9.07 \\
\hline 3 & 12.71 & 9.12 \\
\hline Total & 37.24 & 28.99 \\
\hline Mean & 12.41 & 9.66 \\
\hline SD & 0.506 & 0.806 \\
\hline SE & 0.292 & 0.465 \\
\hline
\end{tabular}

Table 3

\begin{tabular}{|c|c|c|}
\hline \multirow{2}{*}{$\begin{array}{c}\text { Set. } \\
\text { No. }\end{array}$} & \multicolumn{2}{|c|}{$\begin{array}{c}\text { Terminalia arjuna plant } \\
\text { Cocoon Wt. in gram }\end{array}$} \\
\cline { 2 - 3 } & Female & Male \\
\hline 1 & 10.16 & 7.69 \\
\hline 2 & 10.38 & 8.58 \\
\hline 3 & 10.22 & 8.6 \\
\hline Total & 30.76 & 24.87 \\
\hline Mean & 10.25 & 8.29 \\
\hline SD & 0.092 & 0.424 \\
\hline SE & 0.053 & 0.244 \\
\hline
\end{tabular}

Table 1,2,3 : Total mean of Female and Male Cocoon weight of 5th instar in the arjuna food plant.In the table 1. Female cocoon mean weight $11.76 \mathrm{gm}$, male mean weight $8.97 \mathrm{gm}$. Table 3 .Female cocoon mean weight $12.4 \mathrm{gm}$ and male cocoon mean weight 9.6gm. In the Table -2. mean weight of female cocoon from Arjuna plant is $12.41 \mathrm{gm}$ and the mean of male cocoon of Arjuna plant is $9.66 \mathrm{gm}$. table 3. We observed that mean weight of Female cocoon from Arjuna plant is $10.25 \mathrm{gm}$ and the mean of Male cocoon of Arjuna plant is $8.29 \mathrm{gm}$,

\section{$1^{\text {st }}, 2^{\text {nd }}, 3$ rd Crop-Pupa weight, table $(4,5,6)$}

Table 4

\begin{tabular}{|c|c|c|}
\hline \multirow{2}{*}{$\begin{array}{c}\text { Set. } \\
\text { No. }\end{array}$} & \multicolumn{2}{|c|}{$\begin{array}{c}\text { Terminalia arjuna plant } \\
\text { Pupa Wt. in gram }\end{array}$} \\
\cline { 2 - 3 } & Female & Male \\
\hline 1 & 11.17 & 9.70 \\
\hline 2 & 9.48 & 7.33 \\
\hline 3 & 10.19 & 6.11 \\
\hline Total & 30.84 & 23.14 \\
\hline Mean & 10.28 & 7.71 \\
\hline SD & 0.692 & 1.49 \\
\hline SE & 0.399 & 0.86 \\
\hline
\end{tabular}

Table 5

\begin{tabular}{|c|c|c|}
\hline \multirow{2}{*}{$\begin{array}{c}\text { Set. } \\
\text { No. }\end{array}$} & \multicolumn{2}{|c|}{$\begin{array}{c}\text { Terminalia arjuna plant } \\
\text { Pupa Wt. in gram }\end{array}$} \\
\cline { 2 - 3 } & Female & Male \\
\hline 1 & 10.06 & 9.01 \\
\hline 2 & 11.23 & 8.03 \\
\hline 3 & 11.13 & 8.01 \\
\hline Total & 32.42 & 25.05 \\
\hline Mean & 10.81 & 8.35 \\
\hline SD & 0.529 & 0.405 \\
\hline SE & 0.305 & 0.233 \\
\hline
\end{tabular}

Table 6

\begin{tabular}{|c|c|c|}
\hline \multirow{2}{*}{$\begin{array}{c}\text { Set. } \\
\text { No. }\end{array}$} & \multicolumn{2}{|c|}{$\begin{array}{c}\text { Terminalia arjuna plant } \\
\text { Pupa Wt. in gram }\end{array}$} \\
\cline { 2 - 3 } & Female & Male \\
\hline 1 & 9.15 & 6.6 \\
\hline 2 & 9.21 & 7.5 \\
\hline 3 & 9.2 & 7.5 \\
\hline Total & 27.56 & 21.6 \\
\hline Mean & 9.19 & 7.2 \\
\hline SD & 0.09 & 0.04 \\
\hline SE & 0.052 & 0.244 \\
\hline
\end{tabular}

In the Table 4 ,5,6. Total mean of Female and Male pupa weight of 5th instar in the arjuna food plant. Table -4. mean weight of female pupa from Arjuna plant is $10.28 \mathrm{gm}$ and the mean of male pupa of Arjuna plant is $7.71 \mathrm{gm}$ In the Table -5. mean weight of female pupa from Arjuna plant is $10.81 \mathrm{gm}$ and the mean of male pupal of Arjuna plant is $8.35 \mathrm{gm}$. Table 6. We observed that mean weight of female pupa from Arjuna plant is $9.19 \mathrm{gm}$ and the mean of male pupa of Arjuna plant is $7.2 \mathrm{gm}$. In the

$1^{\text {st }}, 2^{\text {nd }}, 3$ rd Crop- Shell weight, table $(7,8,9)$

Table 7

\begin{tabular}{|c|c|c|}
\hline \multirow{2}{*}{$\begin{array}{c}\text { Set. } \\
\text { No. }\end{array}$} & \multicolumn{2}{|c|}{$\begin{array}{c}\text { Terminalia arjuna plant } \\
\text { Shell Wt. in gram }\end{array}$} \\
\cline { 2 - 3 } & Female & Male \\
\hline 1 & 1.54 & 1.26 \\
\hline 2 & 1.39 & 1.1 \\
\hline 3 & 1.52 & 1.08 \\
\hline Total & 4.45 & 3.44 \\
\hline Mean & 1.48 & 1.14 \\
\hline SD & 0.066 & 0.072 \\
\hline SE & 0.038 & 0.041 \\
\hline
\end{tabular}

Table 8

\begin{tabular}{|c|c|c|}
\hline \multirow{2}{*}{$\begin{array}{c}\text { Set. } \\
\text { No. }\end{array}$} & \multicolumn{2}{|c|}{$\begin{array}{c}\text { Terminalia arjuna plant } \\
\text { Shell Wt. in gram }\end{array}$} \\
\cline { 2 - 3 } & Female & Male \\
\hline 1 & 1.64 & 1.07 \\
\hline 2 & 1.6 & 1.04 \\
\hline 3 & 1.5 & 1.15 \\
\hline Total & 4.74 & 3.26 \\
\hline Mean & 1.58 & 1.09 \\
\hline SD & 0.098 & 0.097 \\
\hline SE & 0.056 & 0.056 \\
\hline
\end{tabular}

Table 9

\begin{tabular}{|c|c|c|}
\hline \multirow{2}{*}{$\begin{array}{c}\text { Set. } \\
\text { No. }\end{array}$} & \multicolumn{2}{|c|}{$\begin{array}{c}\text { Terminalia arjuna plant } \\
\text { Shell Wt. in gram }\end{array}$} \\
\cline { 2 - 3 } & Female & Male \\
\hline 1 & 1.01 & 1.09 \\
\hline 2 & 1.17 & 1.08 \\
\hline 3 & 1.02 & 1.1 \\
\hline Total & 3.2 & 3.27 \\
\hline Mean & 1.06 & 1.09 \\
\hline SD & 0.073 & 2.581 \\
\hline SE & 0.042 & 1.49 \\
\hline
\end{tabular}


.In the Table 7,8,9 .Total mean of Female and Male Shell weight of 5th instar in the arjuna food plant. In the Table -7 mean weight of female shell from Arjuna plant is $1.48 \mathrm{gm}$ and the mean of male shell of Arjun plant is $1.14 \mathrm{gm}$. In the Table $\mathbf{- 8}$ mean weight of female shell from Arjuna plant is $1.58 \mathrm{gm}$ and the mean of male shell of Arjuna plant is 1.09. Table -9. We observed that mean weight of female shell from Arjuna plant is $1.06 \mathrm{gm}$ and the mean of male shell of Arjuna plant is $1.09 \mathrm{gm}$.

Table 1. Data showing I,II and III crop on Terminalia arjuna,cocoon weight $(\mathrm{g})$,Pupa weight $(\mathrm{g}), \operatorname{shell}$ weight $(\mathrm{g})$ of Antheraea mylitta (male)Values are mean+_SE $(\mathrm{N}=3)$

\begin{tabular}{|c|c|c|c|}
\hline crop & Cocoon Weight $(\mathrm{g})$ & Pupa weight (g) & Shell weight (g) \\
\hline I & $\begin{array}{c}8.97 \pm 0.91 \\
\text { (3) }\end{array}$ & $\begin{array}{c}7.71 \pm 0.86 \\
(3)\end{array}$ & $\begin{array}{c}1.14 \pm 0.04 \\
\text { (3) }\end{array}$ \\
\hline II & $\begin{array}{c}9.60 \pm 0.46 \\
\text { (3) }\end{array}$ & $\begin{array}{c}8.30 \pm 0.23 \\
\text { (3) }\end{array}$ & $\begin{array}{c}1.00 \pm 0.05 \\
\text { (3) }\end{array}$ \\
\hline III & $\begin{array}{c}8.29 \pm 0.24 \\
\text { ( 3) }\end{array}$ & $\begin{array}{c}7.20 \pm 0.24 \\
\text { (3) }\end{array}$ & $\begin{array}{c}1.09 \pm 1.49 \\
\text { (3) }\end{array}$ \\
\hline
\end{tabular}

$\mathrm{Xp}<1.49$ in respect to I crop

Table 2. Data showing I,II and III crop on Terminalia arjuna,cocoon weight $(\mathrm{g})$, Pupa weight $(\mathrm{g})$, shell weight $(\mathrm{g})$ of Antheraea mylitta (Female)Values are mean $\pm \mathrm{SE}(\mathrm{N}=3)$

\begin{tabular}{|c|c|c|c|}
\hline crop & Cocoon Weight $(\mathrm{g})$ & Pupa weight (g) & Shell weight $(\mathrm{g})$ \\
\hline $\mathrm{I}$ & $\begin{array}{c}11.76 \pm 0.43 \\
\text { (3) }\end{array}$ & $\begin{array}{c}10.28 \pm 0.39 \\
\text { (3) }\end{array}$ & $\begin{array}{c}1.48 \pm 0.03 \\
\text { (3) }\end{array}$ \\
\hline II & $\begin{array}{c}12.40 \pm 0.29 \\
\text { (3) }\end{array}$ & $\begin{array}{c}10.80 \pm 0.30 \\
\text { (3) }\end{array}$ & $\begin{array}{c}1.50 \pm 0.05 \\
\text { (3) }\end{array}$ \\
\hline III & $\begin{array}{c}10.25 \pm 0.05 \\
\text { ( } 3)\end{array}$ & $\begin{array}{c}9.10 \pm 0.05 \\
(3)\end{array}$ & $\begin{array}{c}1.06 \pm 0.04 \\
(3)\end{array}$ \\
\hline
\end{tabular}

$\mathrm{Xp}<0.04$ in respect to I crop

Ist crop Antheraea mylitta- Feed in Terminalia arjuna

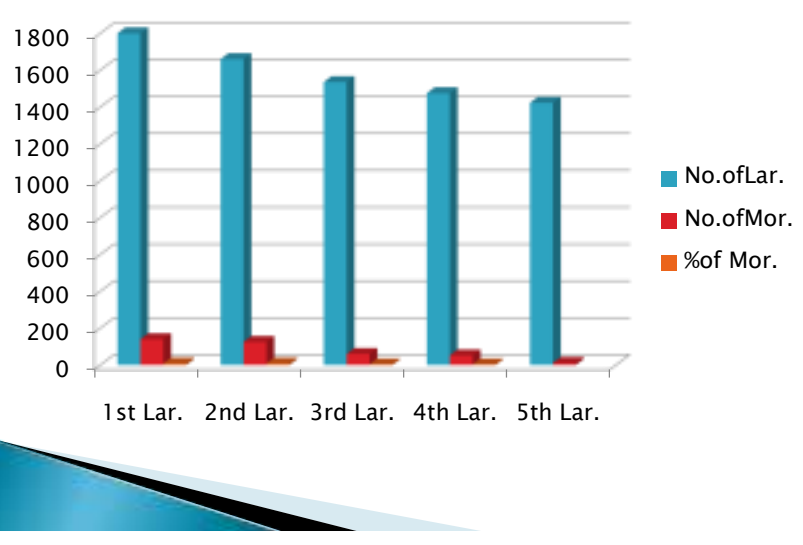

Fig 1 : Graph showing :Effective Rate of Rearing(No.of Larvae 01800)and effective mortality rate

\section{$2^{\text {nd }}$ crop Antheraea mylitta- Feed in Terminalia arjuna}

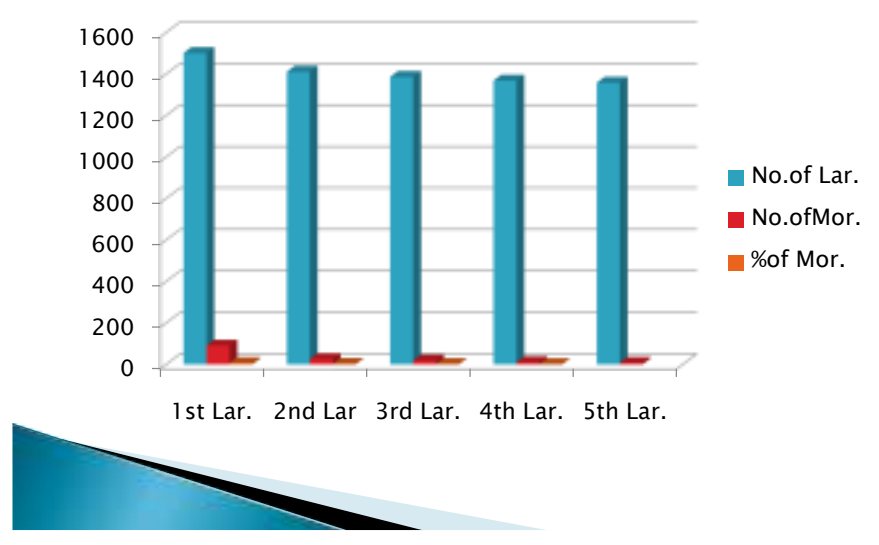

Fig 2 : Graph showing :Effective Rate of Rearing(No.of Larvae 01600)and effective mortality rat 


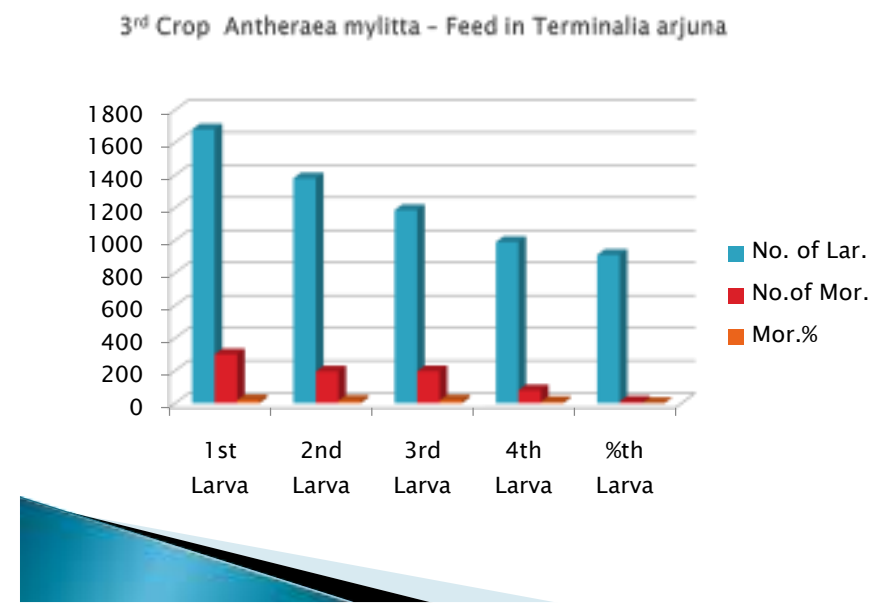

Fig 3: Graph Showing:Effective Rate of Rearing(No.of Larvae 0-1800 and Mortality Rate)

\section{Results and discussion -}

The result of the present study gives insight on the role of different environmental factors on the survivability of Tasar silkworm larvae in different stages in Ambikapur sericulture department.The larval mortality due to bacteria,verious,pestes was recorded in different larval stages in plot.The data analysis that larval mortality rate increases with fluctuation in the temperature and relative humidity existing in the previous two or three days.the environmental factor which exists during 4 days earlier plays a major role in deciding the survivability of the larvae outdoor condition. The results of the present study confirms that more larval mortality was noticed in depend favorable or non favorable environmental condition responses for spread of the diseases. Then main responses of silk production of environmental reasons. After study of doing analysis to find how much contribution of male is in the production of Tasar silkworm.How much contribution of female is also shown in the study report.In theproduction of silk how much directly or indirectly between. The both male and female is concerned is also mentioned is the report.Hence for more silk production depends upon suitable seasons.As a result of production of cocoon can be increased and more and more income can be earned in the production of Tasar silk.

\section{References}

1.chandrakanth N; Moorthy SM.Kariyapa,Ponnuvel KM,Sivaprasad V. Reeling performances of F2 and back cross populations under high Temperature conditions Journal of Entomology and zoology studies 2015;3(6):219-222.

2.RanjanA,Poddar A, Roy Sp, Environmental controlling factors of Tasar silkworm Antheraea mylitta Drury(Lepidoptera:Saturnide), our nature 2012;10:115-118.

3. V.K. Rahmathulla;’Management of climatic factors during silkworm rearing the textile Industry and trade Journal pp.25-26;1999.

4. T.Singh,M.M.Bhat,and M.K.Ashraf,'Insect adaptations to changing Environments temperature and humidity.”International Journal of Industrial Entomology,Vol;19 no.1 pp.'55-165'2009.

5. Dewangan, SK, Sahu, KR and Soni,SK (2012).Breaking of poverty though sericulture among the tribe -A.Socio-Economic study of Dharmjaigarh blok of Raigarh Dist.C.G. India:Research Journal of Recent science, 1:371 - 374.

6. Yadav,G.S.(2000)Studies on the association and interation of temperature.Relative humidity and rainfall in Tasar ecosystem of Vidarbha,Bull.Ind.Acad.Ser.,4(1):20-30.

7. Gregory,S. 1914, Rural Labour and sericulture:Typology Stretegies and prospects,Indian J.Ind.Relat 1,365-376.

8. Singh,C.1993, An Economic Analysis of sericulture production in Raigarh district of Madhya Pradesh,Agricultural Economics Research Review,6,52-53.

9. Srivastava, S.Kapoor, R.Thathola, A. Srivastava, R.P. 2003.Mulberry (momsalba) Leaves as human food,a new dimension of sericulture. Int.J.Food Sci.Nutr.54,411-416.

10. Sheela Patel,R.K.Singh and Shweta Sao(2016) Comparative studies of two silkworm species in Raigarh district for high quality yield.International Journal of Development Research Vol.6,10509-10514. 